\title{
Pharmacokinetic-Pharmacodynamic Modeling of the Effectiveness and Safety of Buprenorphine and Fentanyl in Rats
}

\author{
Ashraf Yassen, ${ }^{1}$ Erik Olofsen, ${ }^{2}$ Jingmin Kan, ${ }^{1}$ Albert Dahan, ${ }^{2}$ and Meindert Danhof ${ }^{1,3,4}$
}

Received February 27, 2007; accepted August 20, 2007; published online October 4, 2007

\begin{abstract}
Objective. Respiratory depression is a serious and potentially life-threatening side-effect of opioid therapy. The objective of this investigation was to characterize the relationship between buprenorphine or fentanyl exposure and the effectiveness and safety outcome in rats.

Methods. Data on the time course of the antinociceptive and respiratory depressant effect were analyzed on the basis of population logistic regression PK-PD models using non-linear mixed effects modeling software (NONMEM). The pharmacokinetics of buprenorphine and fentanyl were described by a threeand two-compartment model, respectively. A logistic regression model (linear logit model) was used to characterize the relationship between drug exposure and the binary effectiveness and safety outcome. Results. For buprenorphine, the odds ratios (OR) were 28.5 (95\% CI, 6.9-50.1) and 2.10 (95\% CI, 0.713.49) for the antinociceptive and respiratory depressant effect, respectively. For fentanyl these odds ratios were 3.03 (95\% CI, 1.87-4.21) and 2.54 (95\% CI, 1.26-3.82), respectively.

Conclusion. The calculated safety index $\left(\mathrm{OR}_{\text {antinociception }} / \mathrm{OR}_{\text {respiratory depression }}\right)$ for fentanyl of 1.20 suggests that fentanyl has a low safety margin, implicating that fentanyl needs to be titrated with caution. For buprenorphine the safety index is 13.54 suggesting that buprenorphine is a relatively safe opioid.
\end{abstract}

KEY WORDS: effectiveness; PK-PD; respiratory depression; safety; utility function.

\section{INTRODUCTION}

Worldwide millions of people suffer from severe (uncontrolled) and sometimes incurable pain and are dependent on the daily intake of analgesic drugs to relief their pain. Opioid analgesics are among the most effective agents currently available for treating postoperative and chronic (malignant) pain. Apart from their analgesic actions, all opiates produce side-effects $(1,2)$. Although most of these side effects are mild in nature and have little clinical relevance, respiratory depression is a potentially life-threatening complication of opiate therapy $(3,4)$. Therefore, careful titration of the opioid dose to the desired analgesic effect is essential to avoid respiratory depression (5). This applies particularly to potent opioid analgesics.

Fentanyl is a $\mu$-opioid agonist which is frequently used both in anesthesia and for the treatment of acute and chronic pain (6-8). In pharmacological terms, fentanyl is a highefficacy full agonist at the $\mu$-opioid receptor with steep concentration-response curves $(9,10)$. Due to the relatively narrow therapeutic window of fentanyl the risk of severe fatal

\footnotetext{
${ }^{1}$ Division of Pharmacology, Gorlaeus Laboratories, Leiden/Amsterdam Center for Drug Research, P.O. Box 9502, 2300 RA, Leiden, The Netherlands.

${ }^{2}$ Department of Anesthesiology, Pain and Anesthesia Research Unit, Leiden University Medical Center, Leiden, The Netherlands. ${ }^{3}$ LAP\&P Consultants BV, Leiden, The Netherlands.

${ }^{4}$ To whom correspondence should be addressed. (e-mail: m.danhof@ lacdr.leidenuniv.nl)
}

respiratory depression is rather large. As a result fentanyl dosing needs to be carefully titrated to the optimal analgesic effect (11). To facilitate fentanyl dosing, advanced drug delivery systems, aiming at an improved selectivity of action have been introduced. Slow release preparations and transdermal patches for opiates are examples of drug delivery systems which have been developed with the aim to optimize opiate delivery and to reduced side-effects (12).

Another approach towards the reduction of side effects is the use of compounds with a more favorable effectivenesssafety balance. In this respect it is of interest that in theory, partial agonists can have a greater selectivity of action than full agonists (13). Considering the potential lethality of opioid-induced respiratory depression, partial agonists at the $\mu$-opioid receptor provide potential advantages over full agonists, in terms of the effectiveness-safety balance for the treatment of pain. Buprenorphine is a semi-synthetic opioid, which has been in clinical use for the treatment of acute and chronic pain since 1979 (14). Recently, it has been demonstrated that buprenorphine behaves as a partial agonist with respect to respiratory depression both in rats (15) and in humans (16), consistent with its partial agonistic behavior in in vitro bio-assays (17). An important consequence of the observed 'ceiling effect' is that an overdose of buprenorphine is less likely to cause severe and potentially life-threatening respiratory depression. Interestingly, for the antinociceptive effect, buprenorphine displays no ceiling effect in the same concentration range in which maximum respiratory depression is elicited, indicating that within the therapeutic concentration range buprenorphine acts as a full agonist with regard 
to the antinociceptive effect $(18,19)$. Differences in receptor expression in the distinct brain tissues can explain why buprenorphine behaves as partial agonist for one effect (respiratory depression, in brain tissue with no receptor reserve) and as a full agonist for another effect (antinociception, in brain tissue with receptor reserve; 20).

The objective of this study was to characterize the effectiveness and safety of buprenorphine and fentanyl in rats using non-linear mixed effects logistic regression analysis. Therapeutic utility functions (21-24) were constructed to assess the effectiveness/safety balances of buprenorphine and fentanyl.

\section{MATERIALS AND METHODS}

Animal data for the PK-PD analysis of the safety and effectiveness of buprenorphine and fentanyl were obtained from two previously published studies on the PK-PD relationships of buprenorphine and fentanyl for the antinociceptive (18) and respiratory depressant effect (15). The protocols of the investigations were approved by the Ethical Committee on Animal Experimentation of Leiden University.

Briefly, the experimental details of the investigations were as follows. Male Wistar rats (Charles River BV, Zeist, The Netherlands), weighting initially $225-250 \mathrm{~g}$, were used in the studies. Two days before the start of the experiment, indwelling cannulae were implanted in the right femoral artery for the serial collection of blood samples and in the right jugular vein for drug administration. Surgical procedures were performed under anesthesia with an intramuscular injection of $0.1 \mathrm{mg} / \mathrm{kg}$ medetomide hydrochloride (Domitor, $1 \mathrm{mg} / \mathrm{ml}$, Pfizer, Capelle a/d IJssel, The Netherlands) and $1 \mathrm{mg} / \mathrm{kg}$ Ketamine base (Ketalar $50 \mathrm{mg} / \mathrm{ml}$, Parke-Davis, Hoofddorp, The Netherlands).

Different doses of buprenorphine $(0.025$ to $0.1 \mathrm{mg} / \mathrm{kg})$ and fentanyl $(0.015-0.04 \mathrm{mg} / \mathrm{kg})$ were tested to characterize the concentration-antinociceptive effect relationships. For respiratory depression, buprenorphine and fentanyl were tested in the dose ranges $0.025-0.3 \mathrm{mg} / \mathrm{kg}$ and $0.03-0.085 \mathrm{mg} / \mathrm{kg}$, respectively. Buprenorphine and fentanyl were administered via a zero-order intravenous infusion (infusion duration 20-40 min). Antinociception or respiratory depression was measured at pre-defined time points. Serial arterial blood samples were collected at fixed time-points. $50 \mu \mathrm{l}$ plasma was separated from the blood by centrifugation and the plasma samples were stored at $-20^{\circ} \mathrm{C}$ prior to analysis.

\section{Measurement of Antinociception}

A tail flick analgesia meter (Columbus Instruments, Columbus, Ohio, USA) was used to determine the pain sensitivity in the control and the drug-treated rats. Radiant heat was applied using a shutter-controlled lamp as a heat source focused on a spot located 6.5 to $7.5 \mathrm{~cm}$ from the tip of the tail. The intensity of the beam was set at a level producing basal latency times between 2.5 and $3.5 \mathrm{~s}$. To prevent tissue injury, the cut-off time was fixed at $10 \mathrm{~s}$. A digital response time indicator with a resolution of $0.1 \mathrm{~s}$ measured the time between activation of the light beam and the tail flick.

\section{Measurement of Respiratory Depression}

Respiratory depression was determined in unrestrained, conscious animals using whole-body plethysmography for the quantification of ventilation (Model PLY3223, Buxco Electronics Inc., UK). Briefly, the animals were placed in a plethysmograph, consisting of a measurement chamber and an integrated reference chamber to correct for atmospheric disturbances. Both chambers were connected to a differential pressure transducer (TRD5700, Buxco Electronics Inc., UK). In each animal the effects of opiates and vehicle on ventilation were assessed at an inhaled concentration of $6.5 \%$ carbon dioxide on a background of normoxia $(20 \%$ oxygen). The inhalation of the gas mixture lasted $5 \mathrm{~min}$ to ensure that steady-state ventilation had been reached. Tidal volume $\left(V_{\mathrm{T}}\right)$, breathing frequency $(\mathrm{RR})$ and minute ventilation $\left(V_{\mathrm{i}}\right.$, where $\left.V_{\mathrm{i}}=V_{\mathrm{T}} \times \mathrm{RR}\right)$ were obtained from changes in chamber pressure using a low-pressure differential transducer connected to preamplifier modules (MAX2270, Buxco Electronics Inc., UK). During the experiment body temperature was maintained at $37.5^{\circ} \mathrm{C}$ using heating pads. Body temperature was monitored continuously by radiotelemetry (model RPC-1, Data Sciences International, USA).

\section{Determination of Buprenorphine and Fentanyl Concentrations}

Two different chemical assays were used for the determination of buprenorphine and fentanyl plasma concentration in rats. The details of these assays have been described elsewhere (18). Briefly, for buprenorphine, to $50 \mu \mathrm{l}$ plasma $25 \mu \mathrm{l}$ internal standard (4 $\mu \mathrm{g} / 100 \mathrm{ml}{ }^{2} \mathrm{H}_{4}$-buprenorphine) was added. Subsequently, $25 \mu \mathrm{l}$ concentrated ammonia was added and the samples were extracted by liquid/liquid extraction with $600 \mu$ l tert.-butyl methyl ether (MTBE). The chromatographic system consisted of an Agilent HP 1100 HPLC system (Agilent, Waldbronn, Germany) coupled to an API 4000 LC/MS/MS system (Applied Biosystems, Darmstadt, Germany). Chroma- tography was performed on a precolumn (Metaguard Polaris $3 \mu$ C18A $2 \mathrm{~mm}$, Varian Darmstadt, Germany) guarded Synergi $4 \mu$ Hydro-RP 80A column $75 \times 2$ mm (Phenomenex, Aschaffenburg, Germany). The lower limit of quantification (LLOQ) was $0.047 \mathrm{ng} / \mathrm{ml}$ for buprenorphine. The accuracy ranged from 99.4 to $102.1 \%$. The precision, expressed as coefficient of variation, ranged from 2.2 to $6.4 \%$ for concentrations between 0.14 and $8.9 \mathrm{ng} / \mathrm{ml}$.

For fentanyl, to $50 \mu \mathrm{l}$ plasma $25 \mu \mathrm{l}$ internal standard (14.8 ng/ml ${ }^{2} \mathrm{H}_{5}$-fentanyl) was added. After adding $10 \mu \mathrm{l}$ concentrated ammonia the samples were extracted by liquid/ liquid extraction with $600 \mu$ l tert.-butyl methyl ether (MTBE). The chromatographic system consisted of an Agilent HP 1100 HPLC system (Agilent, Waldbronn, Germany) coupled to an API 3000 LC/MS/MS system (Applied Biosystems, Darmstadt, Germany). Chromatography was performed on a precolumn (Metaguard Polaris $3 \mu \mathrm{C} 18 \mathrm{~A} 2 \mathrm{~mm}$, Varian Darmstadt, Germany) guarded Atlantis C18 column $3 \mu$ $100 \mathrm{~mm} \times 2.1 \mathrm{~mm}$ (Waters, Eschborn, Germany). The lower limit of quantification (LLOQ) was $0.118 \mathrm{ng} / \mathrm{ml}$. The accuracy ranged from 87.0 to $96.1 \%$ and the precision from 1.9 to $4.0 \%$ for concentrations in the range from 0.4 to $50.2 \mathrm{ng} / \mathrm{ml}$. 


\section{PK-PD Modeling Procedure}

The pharmacokinetic and pharmacodynamic parameters of fentanyl and buprenorphine were estimated using nonlinear mixed-effects modeling as implemented in the NONMEM software (version V, level 1.1; 25). For pharmacokinetic analysis, a population analysis approach using the first order conditional estimation method with $\eta-\varepsilon$ interaction (FOCE interaction) was undertaken. For logistic regression pharmacodynamic analysis, the maximum likelihood estimation algorithm in NONMEM with the Laplacian method (FOCE) and the likelihood option was used to estimate the probability of a certain event occurring. All fitting procedures were performed on an IBM-compatible computer (Pentium IV, 1,500 $\mathrm{MHz}$ ) running under Windows XP with the Fortran compiler Compaq Visual Fortan version 6.1. An in-house available S-Plus 6.0 (Insightful Corp., Seattle, WA, USA) interface to NONMEM was used for data processing and management (including tools to perform non-parametric bootstrap) and graphical data display. Model comparisons were based on the objective function values in NONMEM using the likelihood ratio test. The significance level was set at $\alpha=0.01$, which corresponds to a reduction of $6.6 \mathrm{U}$ in objective function value (OFV; $\chi^{2}$ distribution) to discriminate between two nested structural models after inclusion of one additional parameter. Furthermore, the goodness-of-fit of the pharmacodynamic models was evaluated by simulating values of the effectiveness and safety outcome from the raw data. The simulations were repeated a total of a 100 times. The proportions of observed and simulated scores were plotted versus the 25th, 50th (median), 75th quantiles of the pooled opioid biophase concentrations.

\section{Pharmacokinetic Analysis}

One, two and three-compartment linear models were evaluated to characterize the time course of buprenorphine and fentanyl concentration in plasma. The pharmacokinetic parameters were assumed to be log-normally distributed and therefore an exponential distribution model was used to account for inter-individual variability. Proportional error models were used to quantify residual error. Individual Bayesian pharmacokinetic parameter estimates obtained from the final population pharmacokinetic model served as input for pharmacodynamic model to predict fentanyl and buprenorphine concentrations at observation scores time points (two-stage approach). The extent of Bayesian shrinkage was evaluated for each parameter in the final population pharmacokinetic models of buprenorphine and fentanyl by (26):

$$
\text { Shrinkage }=1-\frac{S D_{\eta P K \text { parameter }}}{\Omega_{P K \text { parameter }}}
$$

in which the SD is the standard deviation of the individual estimates of $\eta$ for each pharmacokinetic parameter and $\Omega_{P K \text { parameter }}$ is the estimate of the standard deviation of the estimated population variance.

To quantify the precision of the pharmacokinetic parameters estimates a non-parametric bootstrap was performed.
Briefly, one thousand bootstrap replicates were generated by sampling randomly from the original data set with replacement. Subsequently, the data sets were fitted one at the time to the final population PK model. The mean, standard error, coefficient of variation and the lower and upper $95 \%$ confidence intervals of the model parameter values were calculated and compared to the typical model parameter values from the original study.

\section{Pharmacodynamic Data Analysis}

The effectiveness (antinociception) and safety (respiratory depression) variable were modeled as dichotomous category variables. The following transformation statements were applied to convert the continuous data into logical twovalued data:

- If antinociceptive effect $\geq 10 \mathrm{~s}$, then observation score is 1 (a yes response; complete antinociception) else 0 (a no response; no antinociception)

- If respiratory depressant effect $\geq 50 \%$, then observation score is 1 (a yes response; severe respiratory depression) else 0 (a no response; no respiratory depression)

Logistic regression analysis was performed to determine the relationship between opioid concentration at the biophase $\left(C_{e}\right)$ and its respective antinociceptive or respiratory depressant effect in rats. The opioid biophase concentration was predicted using a standard effect-compartment model (27):

$$
\frac{d C_{e}}{d t}=k_{e 0} \cdot\left(C_{p}-C_{e}\right)
$$

in which $C_{p}$ represents the opioid plasma concentration, $C_{e}$ the opioid concentration at the biophase and $k_{\mathrm{e} 0}$ the first-order rate constant for biophase distribution.

A linear logit model was fitted to relate the probability of having complete antinociception or respiratory depression to drug exposure at the biophase:

$$
P_{\left(Y_{i j}=1\right)}=\frac{1}{1+e^{-\left(f\left(C_{e}\right)\right)}}
$$

The logit transformation ensures that the probability $P$ will fall between 0 and $1, f\left(C_{e}\right)$ is a linear function of drug exposure at the biophase:

$$
f\left(C_{e}\right)=\alpha+\beta\left(C_{e}\right)
$$

in which $\alpha$ and $\beta$ are the intercept and slope of the regression equation and $C_{e}$ is the drug biophase concentration. Incorporation of individual random effects, $\eta$ with mean zero and variance $\omega^{2}$, was tried by assuming exponential distributions for the regression parameters ( $\alpha$ and $\beta$ ). For each concentrationeffect relationship, the odds ratio was calculated using the exponent of the estimated slope $\beta\left(e^{\beta}\right)$. The odds ratio is defined as the change in the estimated odds of the outcome when the biophase concentration increases by $1 \mathrm{U}$. Bootstrap analysis was performed with the final logistic regression pharmacodynamic models to construct the $95 \%$ confidence intervals of the calculated odds ratios. 
Table I. Parameter Estimates (95\% CI) for the Final Population Pharmacokinetic Model of Buprenorphine

\begin{tabular}{lccccc}
\hline & \multicolumn{2}{c}{ Original Data } & & \multicolumn{2}{c}{1,000 Bootstrap Replicates } \\
\cline { 2 - 3 } \cline { 5 - 6 } & Typical Estimate & Inter-Animal Variability $\omega^{2}$ & & Typical Estimate & Inter-Animal Variability $\omega^{2}$ \\
\hline $\mathrm{Cl}(\mathrm{ml} / \mathrm{min})$ & $18(16.3-19.7)$ & $0.128(0.108-0.148)$ & & $18(16.1-19.9)$ & $0.125(0.086-0.164)$ \\
$V_{1}(\mathrm{ml})$ & $110(77-142)$ & $0.936(0.348-1.52)$ & & $108(65-150)$ & $0.914(0.267-1.56)$ \\
$V_{2}(\mathrm{ml})$ & $370(312-428)$ & $0.274(0.117-0.431)$ & & $400(320-480)$ & $0.353(-0.041-0.745)$ \\
$V_{3}(\mathrm{ml})$ & $1,200(1,009-1,391)$ & $0.337(0.200-0.474)$ & & $1,181(907-1,455)$ & $0.239(0.063-0.415)$ \\
$Q_{2}(\mathrm{ml} / \mathrm{min})$ & $30(26-34)$ & - & $30.3(26-35)$ & - \\
$Q_{3}(\mathrm{ml} / \mathrm{min})$ & $11(9.6-12.4)$ & $0.172(0.094-0.250)$ & & $10.2(7.8-12.6)$ & $0.128(-0.048-0.304)$ \\
Proportional error $(\%)$ & $0.032(0.022-0.042)$ & & & & $0.033(0.021-0.045)$ \\
\hline
\end{tabular}

Finally, an overall ordinal utility function was constructed to evaluate the effectiveness/safety balance of buprenorphine and fentanyl as a function of drug exposure. The utility function was determined by the sum of the weighted probabilities of an efficacious and safe outcome. The effectiveness and safety variables were weighted equally. For the desirable antinociceptive effect the weight factor was positive and for respiratory depression the factor was negative. The effectiveness/safety balance, characterized by the utility function was thus expressed as $\operatorname{Pr}\{$ effectiveness $\}-\operatorname{Pr}\{$ safety\}, where $\operatorname{Pr}\{\mathrm{x}\}$ is the probability of outcome $x$ (28). A negative value of the utility function indicates that the respiratory depressant effect dominates and a positive value suggests that the desired antinociceptive effect dominates.

\section{RESULTS}

\section{Pharmacokinetics}

A three-compartment model adequately described the time course of the concentration profiles of buprenorphine after intravenous administration in rats. Inter-individual variability was estimated for all pharmacokinetic parameters except for $Q_{3}$. The parameter estimates with their corresponding standard error (SE) are summarized in Table I. For fentanyl, a two-compartment pharmacokinetic model with inter-animal variability on all pharmacokinetic parameters best described the disposition in plasma. The parameter estimates with their corresponding standard error (SE) are summarized in Table II. Estimates of shrinkage were low and ranged from $0.02(\mathrm{Cl})$ to $0.19\left(V_{1}\right)$ for buprenorphine and from $0.03(\mathrm{Cl})$ to $0.12\left(V_{2}\right)$ for fentanyl, suggesting that the individual pharmacokinetic parameter estimates of buprenorphine and fentanyl are robust. For comparison, values of shrinkage close to 1 indicate poor individual estimates of the pharmacokinetic parameters. The goodness-of-fit plots (predicted/individual predicted versus observed concentrations, population predicted concentrations versus weighted residuals for population predictions (WRES) and time versus weighted residuals for population predictions (Figs. 1 and 2) indicated that the pharmacokinetic models adequately described the concentrations of buprenorphine and fentanyl. Finally, bootstrap analysis showed that the 95\% CI of the pharmacokinetic parameter estimates and their random effect variables, except for $\omega^{2} V_{2}$ of buprenorphine, did not overlap their respective null values (Tables I and II).

\section{Pharmacodynamics}

After transformation of the continuous data into categories, 615 (35 animals) and 444 (32 animals) observations of response/no response scores to complete antinociception and severe respiratory depression, respectively, were available for analysis of the pharmacodynamic effect of buprenorphine in rats. For fentanyl, 486 (40 animals) and 216 (32 animals) observations were included for pharmacodynamic analysis for the antinociceptive and respiratory depressant effect, respectively. For the antinociceptive effect of buprenorphine, the values of parameters characterizing the linear logistic regression model were $-13.3(95 \%$ CI, -16.08 to -10.52$)$ for the intercept $\alpha$ and 3.35 (95\% CI, 2.59-4.11) for the slope $\beta$ of the regression equation. The odds ratio of complete antinociception occurring is equal to $e^{3.35}$, corresponding to an odds ratio of 28.5 (95\% CI, 6.9-50.1). The odds ratio is statistically different from 1 , indicating that the probability of complete antinociception occurring is dependent on the buprenorphine plasma concentration. Inter-animal variability, $\omega^{2}$ was estimated for $\beta$ and was 0.130 (95\% CI, 0.08-0.181). For respiratory depression, the typical values estimates for

Table II. Parameter Estimates (95\% CI) for the Final Population Pharmacokinetic Model of Fentanyl

\begin{tabular}{lccccc}
\hline & \multicolumn{2}{c}{ Original Data } & & \multicolumn{2}{c}{1,000 Bootstrap Replicates } \\
\cline { 2 - 3 } \cline { 5 - 6 } & Typical Estimate & Inter-Animal Variability $\omega^{2}$ & & Typical Estimate & Inter-Animal Variability $\omega^{2}$ \\
\hline $\mathrm{Cl}(\mathrm{ml} / \mathrm{min})$ & $17.0(16.9-18.1)$ & $0.060(0.040-0.080)$ & & $16.6(15.7-17.4)$ & $0.058(0.038-0.078)$ \\
$V_{1}(\mathrm{ml})$ & $250(218-282)$ & $0.227(0.129-0.325)$ & & $255(222-287)$ & $0.224(0.126-0.322)$ \\
$V_{2}(\mathrm{ml})$ & $440(404-476)$ & $0.080(0.041-0.119)$ & & $444(409-479)$ & $0.077(0.039-0.116)$ \\
$Q(\mathrm{ml} / \mathrm{min})$ & $9.7(8.3-11.1)$ & $0.224(0.126-0.312)$ & & $9.7(8.3-11.1)$ & $0.216(0.118-0.314)$ \\
Proportional error $(\%)$ & $0.034(0.0240 .044)$ & & & & $0.034(0.024-0.044)$ \\
\hline
\end{tabular}




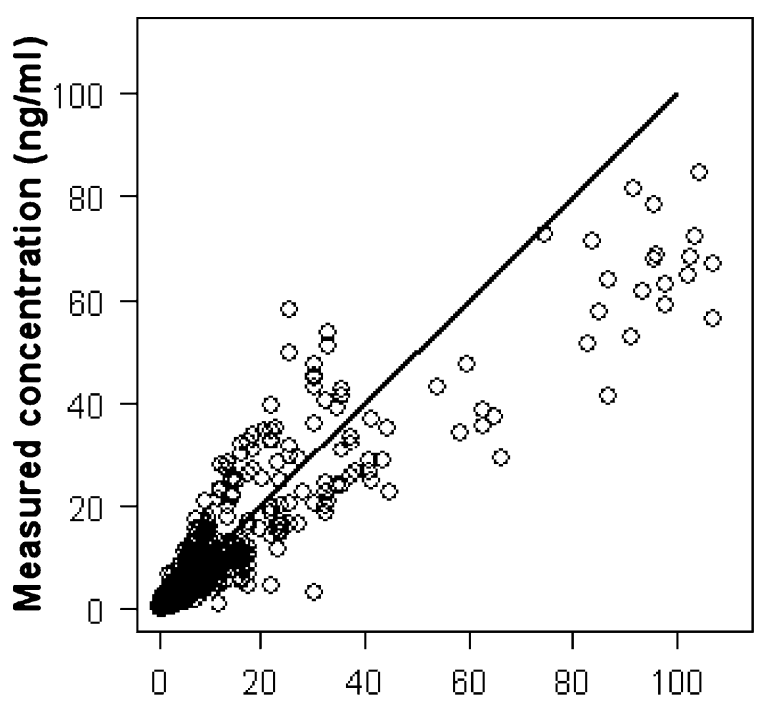

Population predicted concentration (ng/ml)

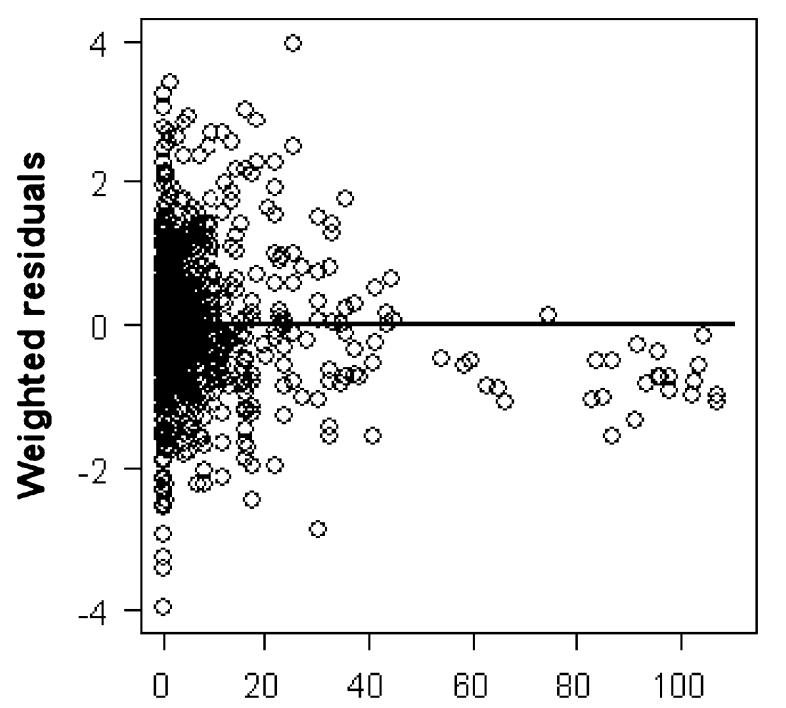

Population predicted concentration ( $\mathrm{ng} / \mathrm{ml}$ )

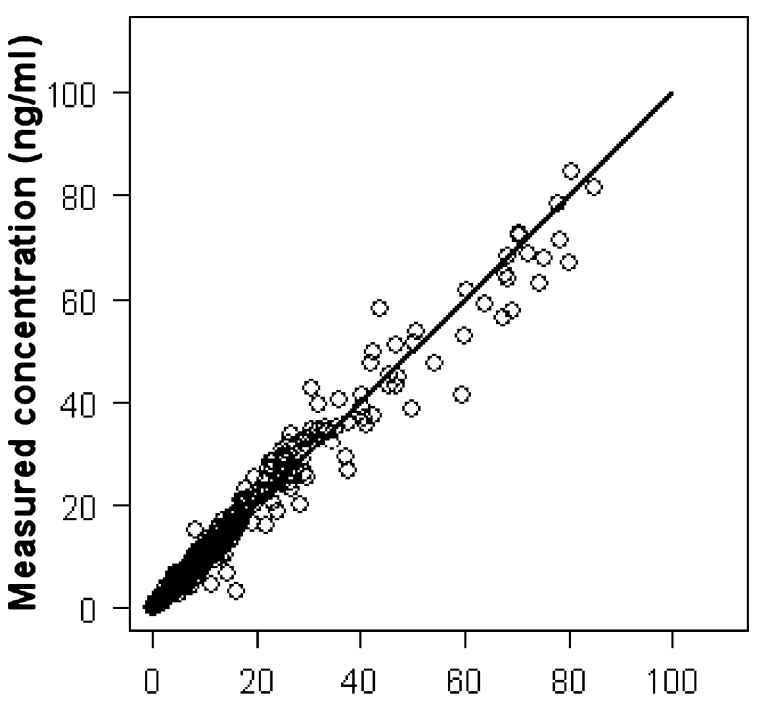

Individual predicted concentration (ng/ml)

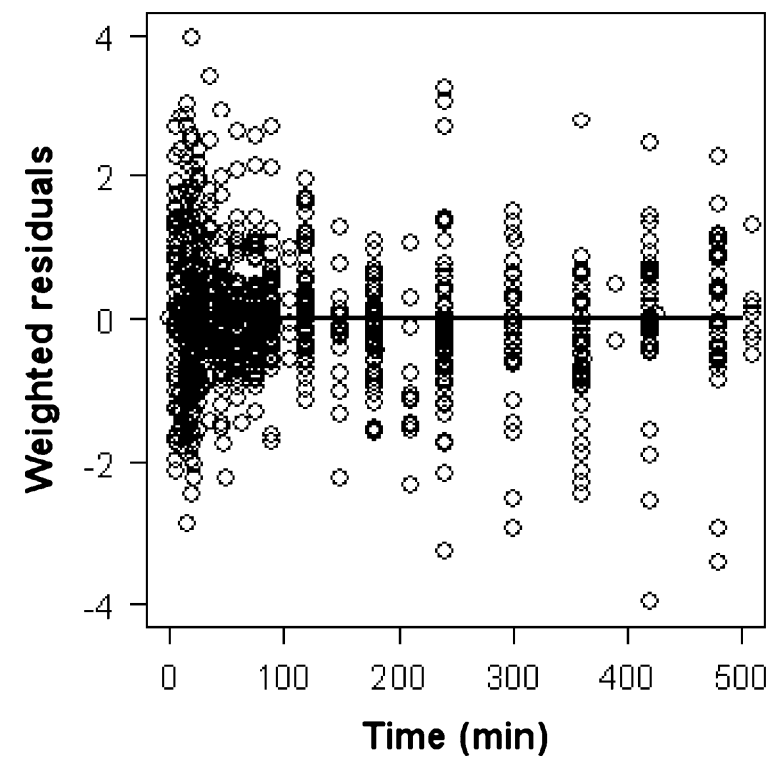

Fig. 1. Goodness-of-fit plots for the final population pharmacokinetic model of buprenorphine.

the intercept $(\alpha)$ and slope $(\beta)$ of the regression equation were -5.22 (95\% CI, -6.54 to -3.90$)$ and $0.744(95 \% \mathrm{CI}$, $0.083-1.405)$, respectively. The odds ratio of severe respiratory depression occurring is equal to $e^{0.774}$, corresponding to an odds ratio of $2.10(95 \% \mathrm{CI}, 0.71-3.49)$. The odds ratio is not statistically different from 1 , indicating that the probability of observing severe respiratory depression does not change within the studied buprenorphine biophase concentration range. Inter-animal variability, $\omega^{2}$ was estimated for $\beta$ and was 1.82 (95\% CI, 0.57-3.07). The odds ratio estimates of buprenorphine obtained from the logistic regression analysis are shown in Table III. Goodness-of-fit was evaluated by the objective function value (OFV) and by simulating the raw data from the final pharmacodynamic logistic regression model. The proportion of the simulated values of the outcome scores for buprenorphine agreed well with the observed scores as can be seen in Fig. 3. For fentanyl, the values of the parameters $\alpha$ and $\beta$ were -8.77 (95\% CI, -11.49 to -6.05$)$ and $1.11(95 \%$ CI, 0.726-1.494), respectively. The odds ratio for the antinociceptive effect was 3.03 (95\% CI, 1.87-4.21) and was significantly different from $1(p<0.05)$. Inter-animal variability, $\omega^{2}$ was estimated for $\beta$ and was 0.0839 (95\% CI, $\left.-0.01-0.177\right)$. For the respiratory depressant effect the values of the logistic regression parameters $\alpha$ and $\beta$ were -5.86 (95\% CI, -8.17 to -3.55$)$ and 0.930 (95\% CI, 0.430-1.430). The odds ratio for the occurrence of severe respiratory depression was $2.54(95 \%$ CI, 1.26 to 3.82$)$. Inter-animal variability, $\omega^{2}$ was estimated for $\beta$ and was 0.193 (95\% CI, 0.085 to 0.300 ). The odds ratio estimates of fentanyl obtained from the logistic regression analysis are shown in Table III. The goodness-of-fit plots for fentanyl are shown in Fig. 4. In the final analysis, the half-time of biophase distribution was included in the final model. 


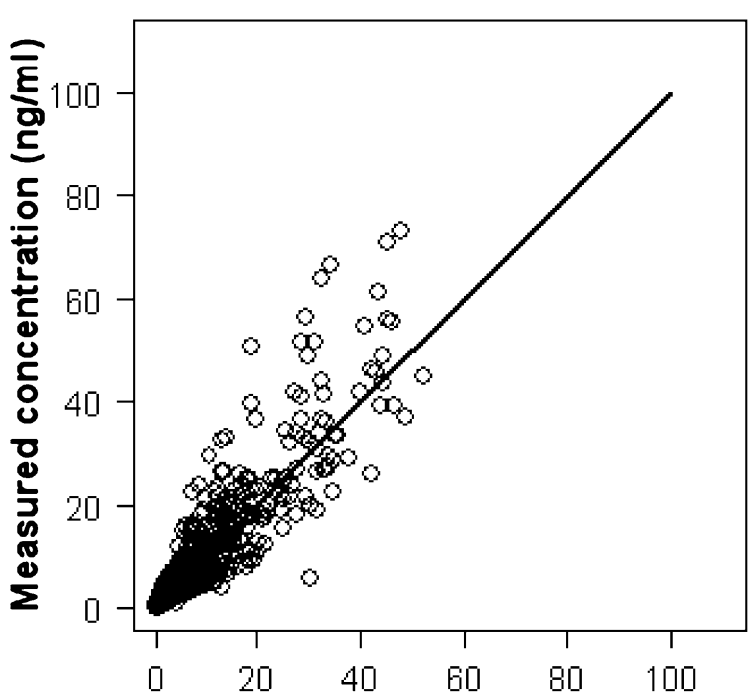

Population predicted concentration ( $\mathrm{ng} / \mathrm{ml}$ )

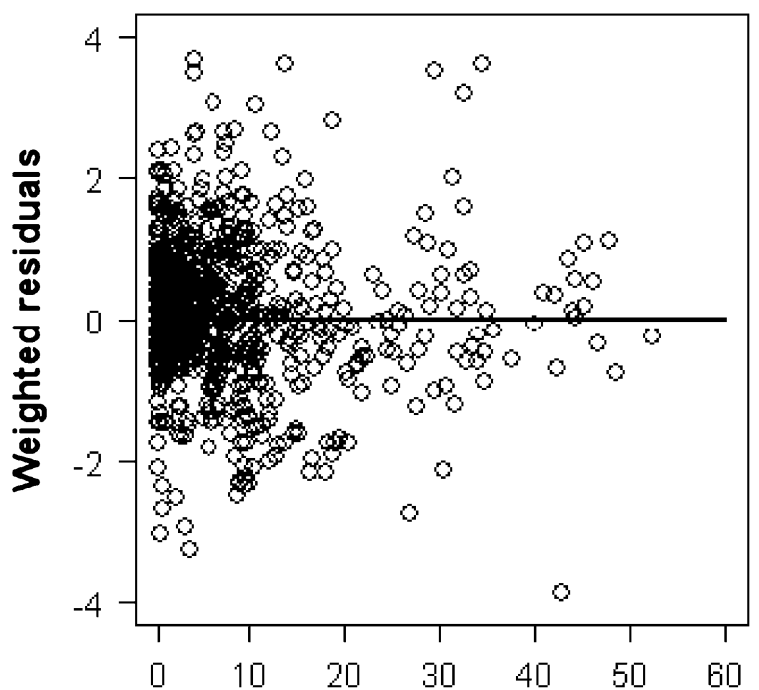

Population predicted concentration ( $\mathrm{ng} / \mathrm{ml}$ )

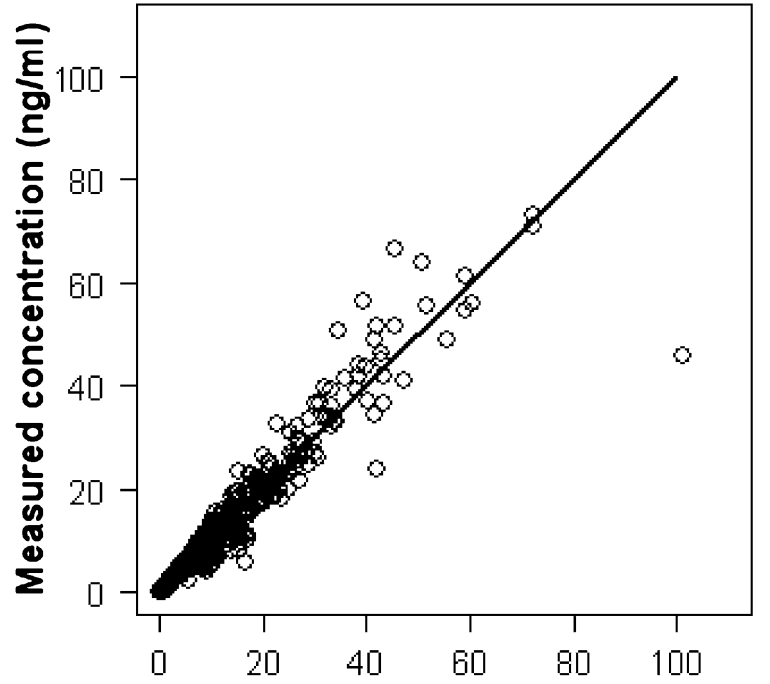

Individual predicted concentration ( $\mathrm{ng} / \mathrm{ml}$ )

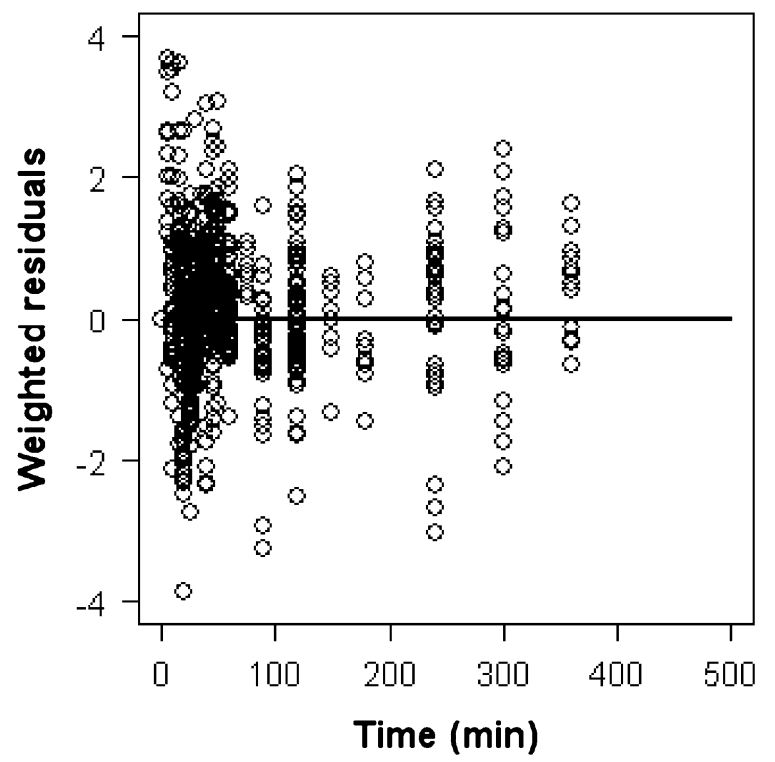

Fig. 2. Goodness-of-fit plots for the final population pharmacokinetic model of fentanyl.

Although its value is not significantly different from zero, its inclusion significantly improved the fits as judged by a significant drop ( $>6.6$ points) in objective function value.

Finally, a therapeutic overall utility function was constructed for the effectiveness/safety balance of buprenorphine and fentanyl. In Fig. 5, it is shown that in the measured concentration range, the utility function of buprenorphine is positive. This indicates that the antinociceptive effectiveness of buprenorphine exceeds the risk of the occurrence of respiratory depression. For fentanyl, the utility function is negative in the measured concentration range, indicating that fentanyl produced more respiratory depression than buprenorphine. At relatively high concentrations the utility function is close to zero for fentanyl and buprenorphine, suggesting that the probability of a desired and side-effect are equal.

\section{DISCUSSION}

Population PK-PD models of buprenorphine and fentanyl, administered intravenously with plasma concentration as the exposure variable and the response to complete antinociception or severe respiratory depression as a binary outcome variable were developed to evaluate the effectiveness and safety of these opiates in rats.

The assignment of observation scores 0 and 1 to the pharmacodynamic safety outcome to indicate "no" or "severe" respiratory depression, respectively, was arbitrarily chosen and is a matter of discussion. Despite its severity, there is no clear and unifying definition for respiratory depression in the literature (29). Partly this is related to the difficulty of measuring opiate-induced respiratory depression in a reliable and strictly quantitative manner. Although 


\section{Observations antinociception}

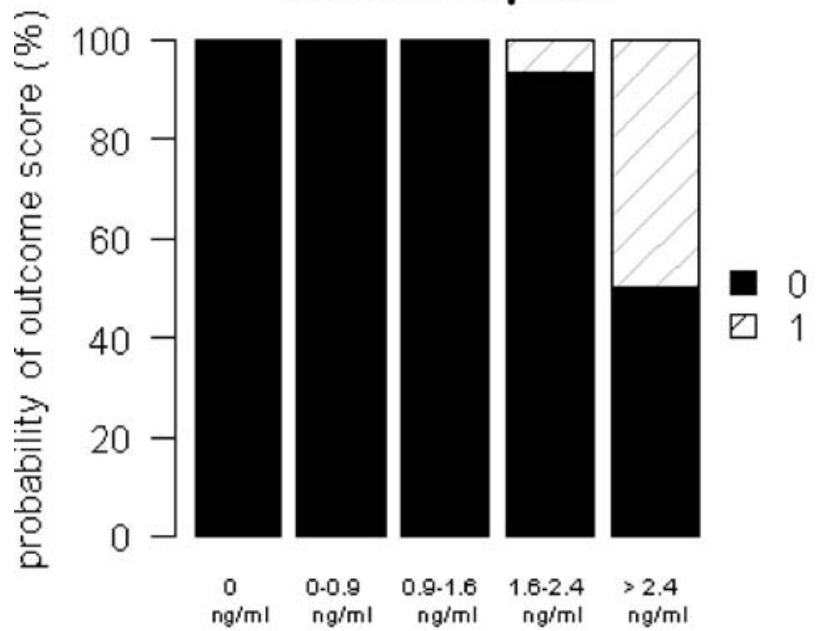

Buprenorphine concentration category

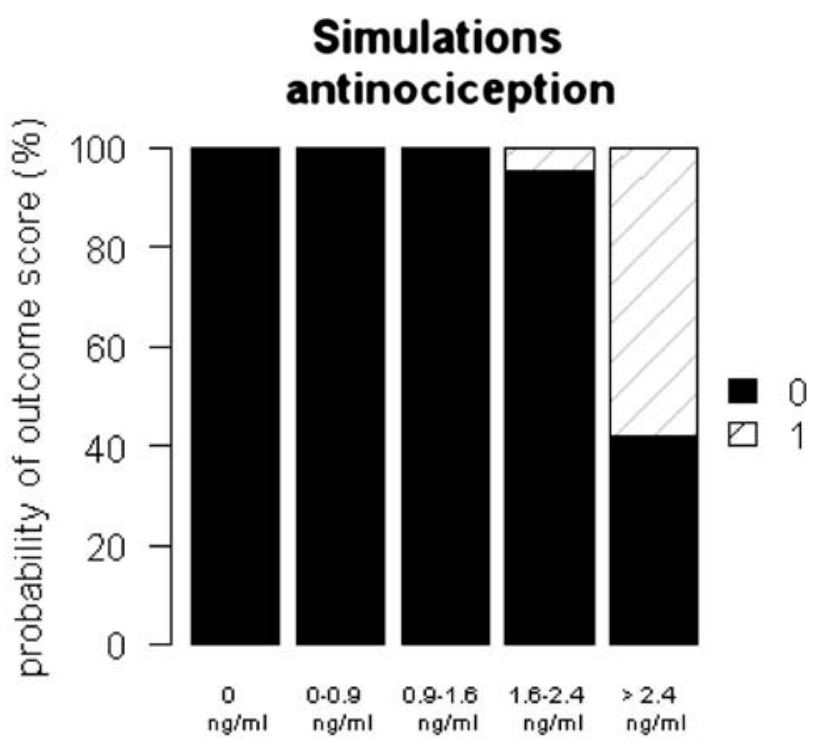

Buprenorphine concentration category

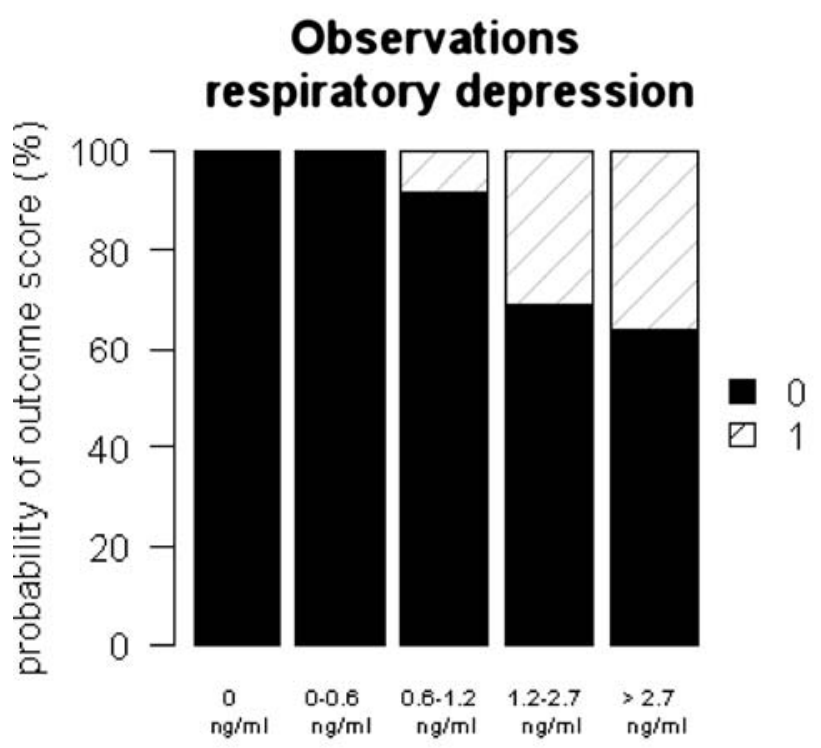

Buprenorphine concentration category

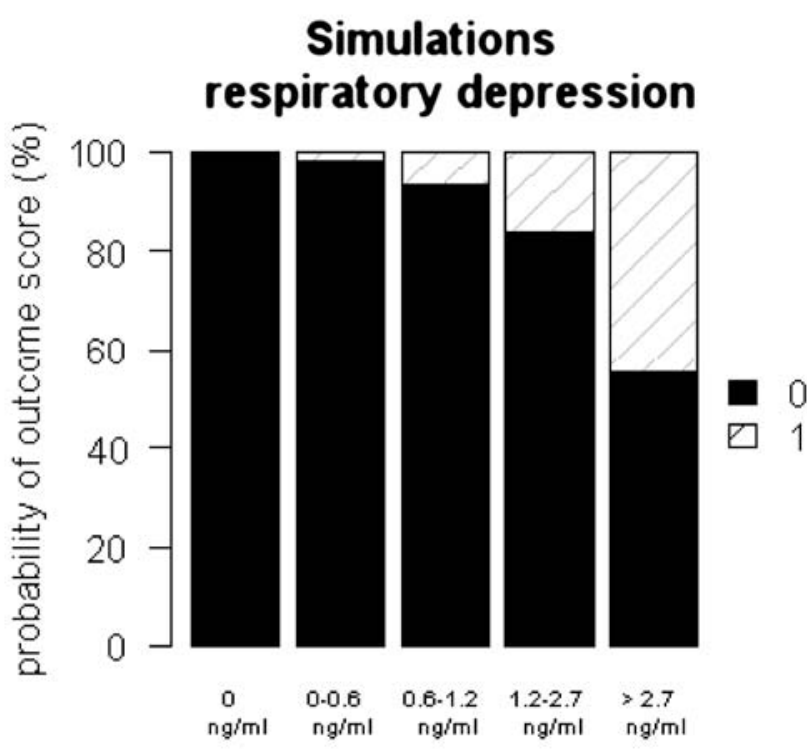

Buprenorphine concentration category

Fig. 3. Goodness-of-fit plots of the logistic regression model for buprenorphine. Observed (left panels) and simulated (right panels) values of the buprenorphine's effectiveness (upper panels) and safety (lower panels) outcome for the different concentration categories. Simulations were repeated in total a 100 times. The concentrations were divided in the following categories (scores at $C_{\mathrm{e}}=0$, between 0 and 25 th quantiles, 25th-50th quantiles, 50th-75th quantiles, $>75$ th quantiles; $\mathbf{s c o r e}=0$ and $\square$ score $=1$ ).

various biomarkers for respiratory depression have been proposed, including the measurement of respiratory rate (30) and arterial $P_{\mathrm{a}} \mathrm{CO}_{2}$ values (31), many of these biomarkers are rather subjective and only provide a qualitative assessment of respiratory depression (32). Furthermore, many of the techniques used to characterize respiratory depression, may well underestimate the respiratory depressant effect of opiates (11). In contrast, minute ventilation measured by whole-body plethysmography or the dynamic end-tidal forcing technique has been shown to be an objective, reproducible and sensitive measure of the respiratory effects of opiates (33). More
Table III. Odds Ratio and Half-Time of Biophase Distribution $\left(T_{1 / 2, \mathrm{ke} 0}\right)$ Estimates $(95 \% \mathrm{CI})$ Obtained From the Logistic Regression Pharmacodynamic Analysis for Buprenorphine and Fentanyl

\begin{tabular}{lcc}
\hline$T_{1 / 2, \mathrm{ke} 0}(\mathrm{~min})$ & Buprenorphine & \multicolumn{1}{c}{ Fentanyl } \\
\hline Antinociception & $86.4(71.3-101.5)$ & $6.08(4.43-7.73)$ \\
Respiratory depression & $109(49-169)$ & $1.97(-0.32-4.26)$ \\
Odds ratio & & \\
Antinociception & $28.5(6.9-50.1)$ & $3.03(1.87-4.21)$ \\
Respiratory depression & $2.10(0.71-3.49)$ & $2.54(1.26-3.82)$ \\
\hline
\end{tabular}




\section{Observations antinociception}

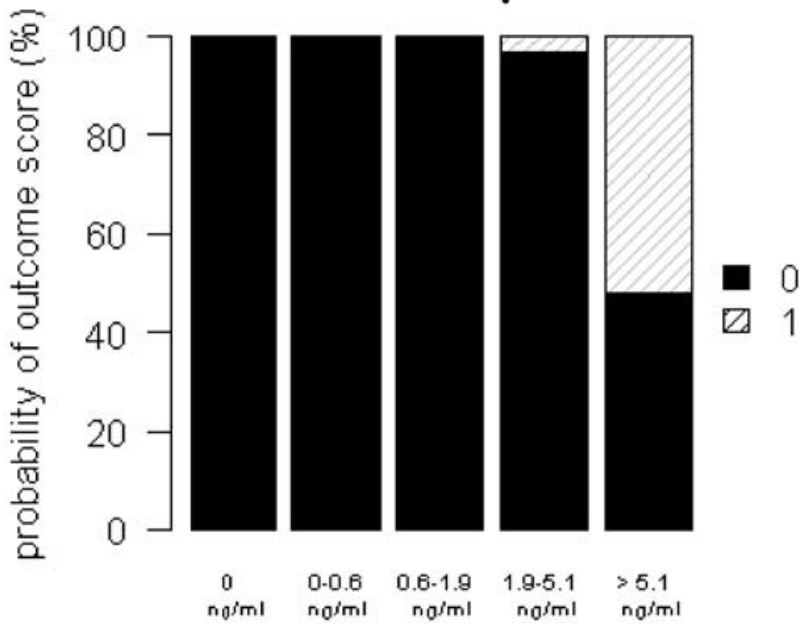

fentanyl concentration category

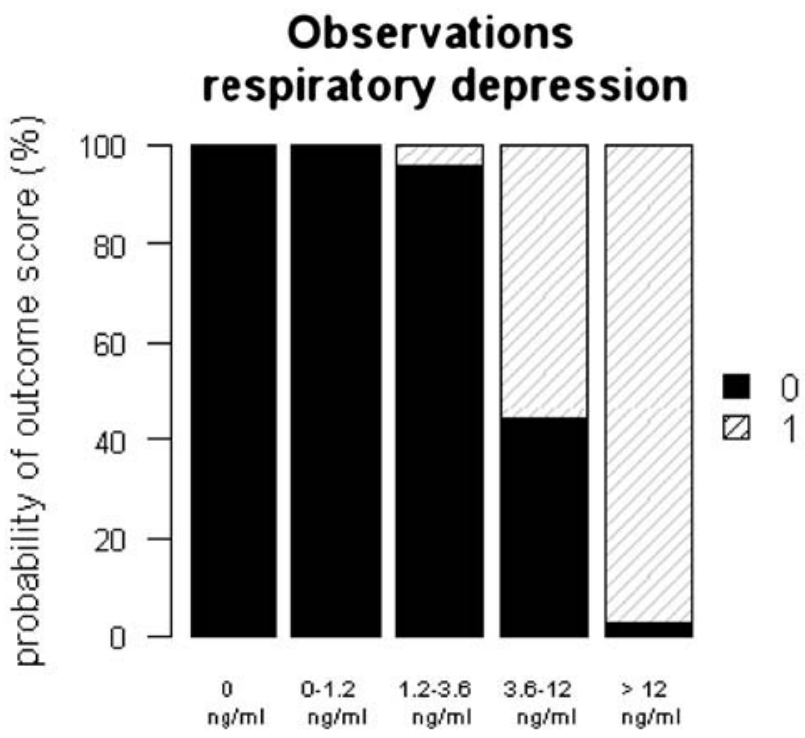

fentanyl concentration category

\section{Simulations antinociception}

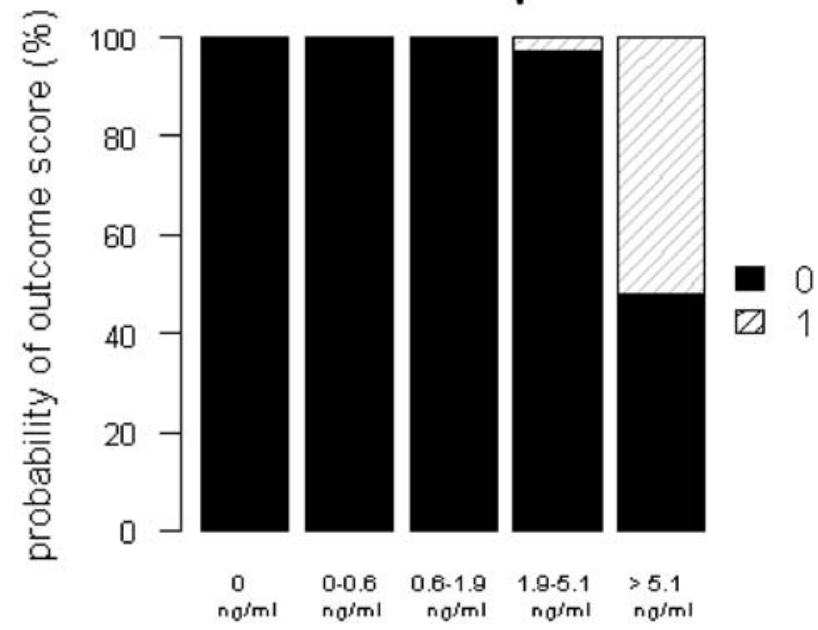

fentanyl concentration category

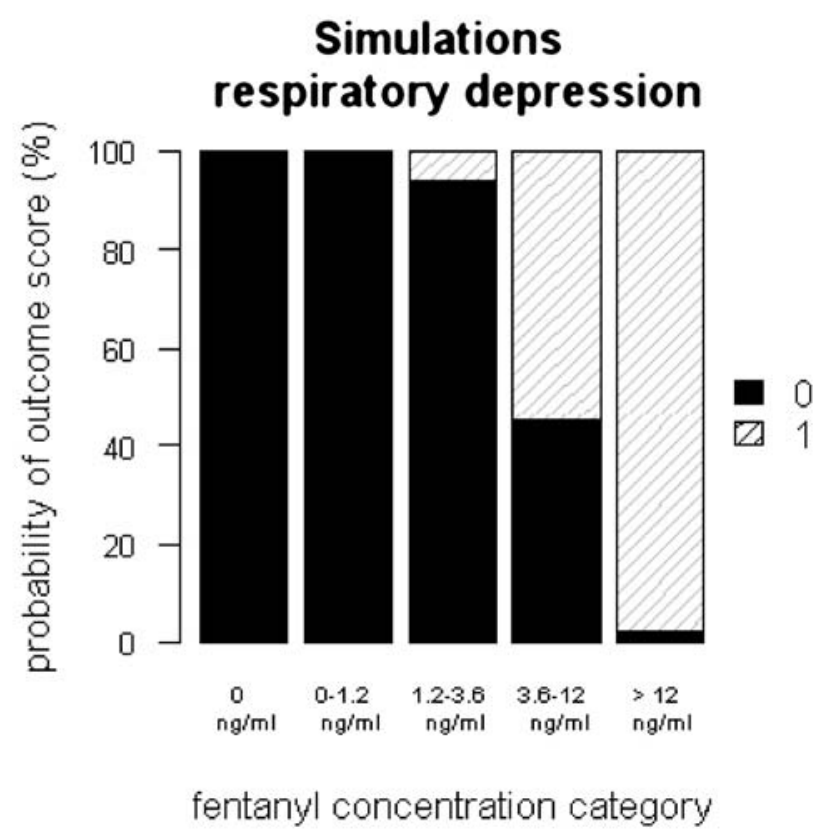

Fig. 4. Goodness-of-fit plots of the logistic regression model for fentanyl. Observed (left panels) and simulated (right panels) values of the buprenorphine's effectiveness (upper panels) and safety (lower panels) outcome for the different concentration categories. Simulations were repeated in total a 100 times. The concentrations were divided in the following categories (scores at $C_{\mathrm{e}}=0$, between 0 and 25 th quantiles, 25 th50th quantiles, 50th-75th quantiles, $>75$ th quantiles; $\mathbf{\text { score }}=0$ and $\square$ score $=1$ ).

importantly, minute ventilation as a biomarker of respiratory depression has been shown to reflect the functioning of various opiates at the $\mu$-opioid receptor in a strictly quantitative manner $(15,16,34)$. Since measurement of minute ventilation allows for a quantitative assessment of respiratory depression, we defined respiratory depression as a decrease in ventilation of more than $50 \%$ relative to baseline. For the antinociceptive effect, reaching the cut-off tail flick latency time is frequently used to indicate that complete antinociception is reached (35). In the present investigation, therefore, the same definition was used to indicate complete antinociception.
PK-PD analysis revealed marked difference in the kinetics of onset and offset for buprenorphine versus fentanyl. The biophase distribution of buprenorphine was significantly slower than for fentanyl. On the basis of the physico-chemical properties (buprenorphine being more lipophilic than fentanyl; 16) it can be expected that buprenorphine displays slower biophase distribution. Furthermore, buprenorphine's slow receptor association/dissociation kinetics contributes to the delay in the onset of buprenorphine's analgesic and respiratory depressant effect. Previously it was shown that the delay in buprenorphine's antinociceptive and 


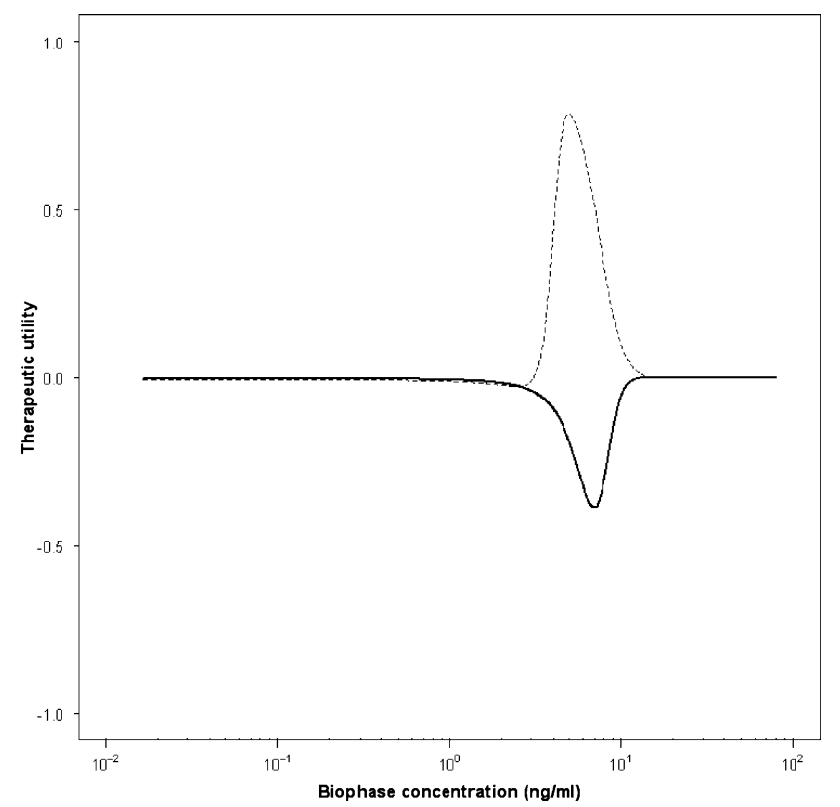

Fig. 5. Estimated therapeutic utility function of buprenorphine (dashed line) or fentanyl (solid line) treatment as a function of its respective biophase exposure. The therapeutic utility function is defined as probability of antinociception-probability of respiratory depression.

respiratory depressant effect is mainly caused by biophase distribution rather than slow receptor association/dissociation kinetics $(15,18)$.

Beside the kinetics of the opioid action, PK-PD analysis yielded important information on the effectiveness and safety outcome of fentanyl and buprenorphine. The odds ratios which had been calculated for the estimated probability of a complete antinociceptive or severe respiratory depressant event can be used as a measure of pharmacological effect size. For fentanyl, the calculated odds ratios for the antinociceptive and respiratory depressant effect were 3.03 (95\% CI, 1.87-4.21) and 2.54 (95\% CI, 1.26-3.82), respectively, suggesting that increasing the fentanyl concentration with $1 \mathrm{ng} / \mathrm{ml}$, there is a 3.03 and 2.54 greater chance of having complete antinociception or respiratory depression, respectively. In terms of probabilities rather than changes in odds, the estimated risk of severe respiratory depression increased from 10.5 to $22.9 \%$ when the concentration was increased from 4 to $5 \mathrm{ng} / \mathrm{ml}$. In addition, the probability of achieving complete antinociception increased from 0.4 to $1.2 \%$. The estimated values of the odds ratio for antinociception (3.03) and respiratory depression (2.54) are nearly identical and suggest that fentanyl displays almost no in vivo tissue selectivity. Accordingly, the ratio of the two OR's (antinociception/respiratory depression) is equal to 1.20 (3.03:2.54) for fentanyl. This ratio can be used as a safety index and suggests that fentanyl displays no selectivity of action.

For buprenorphine, the odds ratios were 28.5 (95\% CI, 6.9-50.1) and 2.10 (95\% CI, 0.71-3.49) for the antinociceptive and respiratory depressant effect, respectively. The large difference in calculated odds ratio's for the antinociceptive and respiratory depressant effect indicates that buprenorphine's displays a varying degree of tissue selectivity. The safety index is equal to 13.54 (28.5:2.10) and suggests a favorable selectivity of action. This provides further evidence, in contrast to fentanyl, for the relative safety of buprenorphine when administered in therapeutic analgesic doses. In various receptor binding studies, buprenorphine has been shown to act as a partial agonist at the $\mu$-opioid receptor (17). Partial agonists typically require complete receptor occupancy for maximal response (36). For the respiratory depressant effect, the in vitro partial agonist activity correlates well its in vivo respiratory depressant effect, characterized by ceiling at higher concentrations $(37,38)$.

Buprenorphine and fentanyl are $\mu$-opioid agonists with distinct pharmacological properties. Since fentanyl behaves as full agonist its in vivo potency for respiratory depression and antinociception are determined by both the affinity and the intrinsic efficacy at the $\mu$-opioid receptor In contrast, for the partial agonist buprenorphine the in vivo potency is determined solely by its affinity for the $\mu$-opioid receptor (39). These differences between efficacy- and affinity-driven agonism may have major implications for the therapeutic utility of opioids. Therapeutic utility is a measure of the effectiveness of drugs. Given this measure, one can speak of increasing or decreasing utility and thereby explain pharmacological behavior of a drug in terms of attempts to optimize pharmacotherapy. As recently discussed by Cullberg et al. $(2005 ; 28)$ the most adequate representation of drug's effectiveness/safety balances with a utility function remains a matter of debate. In the present study, the therapeutic utility function is equal to the sum of the weighted probabilities of an effective (antinociception) and a safety (respiratory depression) outcome. The effect measures, antinociception and respiratory depression, were weighted equally and this seems justified, given that respiratory depression is a potentially life-threatening sideeffect with an acute time course. Furthermore, for the construction of the utility function the continuous antinociceptive and respiratory depressant continuous effect variables were transformed into dichotomous data. This type of data transformation will entail loss of pharmacodynamic information. Especially the kinetics of onset and offset of the opioids effect can be estimated less accurately. However, the kinetics of onset and offset of buprenorphine's antinociceptive and respiratory depressant effect have been characterized previously $(15,18)$. Using a continuous measure of respiratory depression and antinociception one can still construct utility functions. However, using this approach it requires a clear definition of an $E_{\max }$ value for respiratory depression and antinociception. For antinociception no $E_{\max }$ could be identified in the studied dose range. As a consequence the therapeutic utility of buprenorphine can be overestimated when the continuous variables were used for the construction of a utility function. Therefore, the continuous data were transformed into dichotomous data enabling the definition of clear cut-offs.

An intriguing question remains whether the calculated safety margins and therapeutic utilities of buprenorphine and fentanyl are similar in rats and humans. The calculated therapeutic utility is determined by the opioid's affinity and intrinsic effectiveness at the $\mu$-opioid receptor. In theory, these two pharmacological terms are drug-specific parameters, both of which are expected to be similar between man and rat for the $\mu$-opioid receptor (high degree of receptor homology). Recently, it was shown that indeed the drug-specific parameters of buprenorphine are similar in rats and humans for both the antinociceptive and respiratory depressant effect (40). It is therefore advocated that the calculated therapeutic utility for 
buprenorphine, in line with its clinical experience, is similar in rats and humans. Since fentanyl is a full $\mu$-opioid agonist for both antinociception and respiratory depression, producing maximal pharmacological responses in rats and humans, it is expected that the therapeutic utility of fentanyl is not significantly different between these two species. On the basis of the calculated therapeutic utility of fentanyl in rats it can be concluded that fentanyl has a narrow therapeutic window in man (low safety index) requiring fentanyl to be administered cautiously to patients. In fact, a series of recent case-reports on fentanyl-induced severe respiratory depression and death in patients confirm fentanyl's low safety margin underscoring the importance of careful dose titration of high-effectiveness opioids like fentanyl (41). For buprenorphine, the utility function also approaches zero at relatively high concentration upon acute exposure. It should be noted, however, that this is inherent to the definition of the occurrence of respiratory depression in the present study. Previously it has been shown that the maximum respiratory depressant effect of buprenorphine is approximately 48 to $55 \%$ versus 90 to $100 \%$ for fentanyl, suggesting that although the utility function becomes zero, the absolute degree of respiratory depression is less for buprenorphine than for fentanyl. Furthermore, the calculated safety index indicates that buprenorphine has a wider therapeutic window, allowing for the administration of relatively high doses of buprenorphine. Reported safety incidences related to buprenorphine are low and deaths due to buprenorphine misuse are rare. This makes buprenorphine a particularly safe opioid with an increased margin of safety (42).

As evaluation of drug safety becomes increasingly more important in contemporary drug development, there will be a clear shift towards the application of PK/PD modeling to predict drug safety outcomes. As more mechanistic biomarkers become available to assess drug safety, this gives rise to the development of more sophisticated and mechanistic PK/PD models to assess drug effectiveness/safety balances. Clearly, this also influences the selection and structure of therapeutic utility functions to assess the effectiveness/safety balances of drugs under development. To this end, utility function will become less descriptive and more predictive. Also, the potential utility of mechanistic biomarkers to facilitate drug development may lead to better techniques to assess the safety in humans more in line with those used in animals, yielding more accurate animal to human effectiveness/safety extrapolation in an early stage of drug development.

\section{CONCLUSION}

Logistic regression pharmacodynamic models were developed to predict the safety and effectiveness outcome of buprenorphine and fentanyl in rats. Buprenorphine has been shown to display ceiling in respiratory effects but not in analgesia upon acute administration. This explains the relatively high safety index and indicates that the ceiling respiratory effect contributes to the safety profile of buprenorphine. For fentanyl a narrow therapeutic window has been observed, complicating fentanyl dose titration to the optimum analgesic effect without the occurrence of severe respiratory depression. The modeling of the effectiveness/safety balances is a first quantitative assessment of the effectiveness/safety of opiates and these results are in good agreement with the general perception on the safety issues of buprenorphine and fentanyl, underscoring the importance of the development of PK-PD models to quantitatively assess the rationality of pharmacotherapeutic utilities.

\section{REFERENCES}

1. T. A. Bowdle. Adverse effects of opioid agonists and agonistantagonists in anaesthesia. Drug Safety 19:173-189 (1998).

2. T. J. Gan. Risk factors for postoperative nausea and vomiting. Anesth. Analg. 102:1884-1898 (2006).

3. P. L. Bailey, J. K. Lu, N. L. Pace, J. A. Orr, J. L. White, E. A. Hamber, M. H. Slawson, D. J. Crouch, and D. E. Rollins. Effects of intrathecal morphine on the ventilatory response to hypoxia. N. Engl. J Med. 343:1228-1234 (2000).

4. A. D. Baxter. Respiratory depression with patient-controlled analgesia. Can. J. Anaesth. 41:87-90 (1994).

5. C. Lejus, Y. Blanloeil, T. Francois, S. Testa, P. Michel, and B. Dixneuf. Post-operative intravenous continuous analgesia: comparison of buprenorphine, fentanyl and nalbuphine. Eur. J. Anaesthesiol. 13:57-65 (1996).

6. C. Modesti, T. Sacco, G. Morelli, M. G. Bocci, P. Ciocchetti, F. Vitale, V. Perilli, and L. Sollazzi. Balanced anestesia versus total intravenous anestesia for kidney transplantation. Minerva Anestesiol. 72(7-8):627-635 (2006).

7. J. C. Scott, J. E. Cooke, and D. R. Stanski. Electroencephalographic quantitation of opioid effect: comparative pharmacodynamics of fentanyl and sufentanil. Anesthesiology 74:34-42 (1991).

8. K. L. Waara-Wolleat, K. R. Hildebrand, and G. R. Stewart. A review of intrathecal fentanyl and sufentanil for the treatment of chronic pain. Pain Med. 7:251-259 (2006).

9. E. H. Cox, T. Kerbusch, P. H. Van der Graaf, and M. Danhof. Pharmacokinetic-pharmacodynamic modeling of the electroencephalogram effect of synthetic opioids in the rat: correlation with the interaction at the mu-opioid receptor. J. Pharmacol. Exp. Ther. 284:1095-1103 (1998).

10. A. Woodhouse and L. E. Mather. The minimum effective concentration of opioids: a revisitation with patient controlled analgesia fentanyl. Reg. Anesth. Pain Med. 25:259-267 (2000).

11. A. Dahan, A. Yassen, H. Bijl, R. Romberg, E. Sarton, L. Teppema, E. Olofsen, and M. Danhof. Comparison of the respiratory effects of intravenous buprenorphine and fentanyl in humans and rats. Br. J. Anaesth. 94:825-834 (2005).

12. T. L. Skaer. Transdermal opioids for cancer pain. Health Qual. Life Outcomes 4:24 (2006).

13. E. A. Schaickvan, H. E. Tukker, H. C. Roelen, A. P. IJzerman, and M. Danhof. Selectivity of action of 8-alkylamino analogues of N6-cyclopentyladenosine in vivo: haemodynamic versus antilipolytic responses in rats. Br. J. Pharmacol. 124:607-618 (1998).

14. K. Budd and B. J. Collett. Old dog-new (ma)trix. Br. J. Anaesth. 90:722-724 (2003).

15. A. Yassen, J. Kan, E. Olofsen, E. Suidgeest, A. Dahan, and M. Danhof. Mechanism-based pharmacokinetic-pharmacodynamic modeling of the respiratory depressant effect of buprenorphine and fentanyl in rats. J. Pharmacol. Exp. Ther. 319:682-692 (2006).

16. A. Yassen, E. Olofsen, R. Romberg, E. Sarton, L. Teppema, M. Danhof, and A. Dahan. Mechanism-based PK/PD modeling of the respiratory depressant effect of buprenorphine and fentanyl in healthy volunteers. Clin. Pharmacol. Ther. 81:50-58 (2007).

17. P. Huang, G. B. Kehner, A. Cowan, and L. Y. Liu-Chen. Comparison of pharmacological activities of buprenorphine and norbuprenorphine: norbuprenorphine is a potent opioid agonist. J. Pharmacol. Exp. Ther. 297:688-695 (2001).

18. A. Yassen, E. Olofsen, A. Dahan, and M. Danhof. Pharmacokinetic-pharmacodynamic modeling of the antinociceptive effect of buprenorphine and fentanyl in rats: role of receptor equilibration kinetics. J. Pharmacol. Exp. Ther. 313:1136-1149 (2005).

19. A. Yassen, E. Olofsen, R. Romberg, E. Sarton, M. Danhof, and A. Dahan. Mechanism-based pharmacokinetic-pharmacodynamic modeling of the antinociceptive effect of buprenorphine in healthy volunteers. Anesthesiology 104:1232-1242 (2006). 
20. P. H. Van der Graaf, E. A. van Schaick, S. A. Visser, H. J. De Greef, A. P. IJzerman, and M. Danhof. Mechanism-based pharmacokinetic-pharmacodynamic modeling of antilipolytic effects of adenosine A(1) receptor agonists in rats: prediction of tissue-dependent efficacy in vivo. J. Pharmacol. Exp. Ther. 290:702-709 (1999).

21. G. Graham, S. Gupta, and L. Aarons. Determination of an optimal dosage regimen using a Bayesian decision analysis of efficacy and adverse effect data. J. Pharmacokinet. Pharmacodyn. 29:67-88 (2002).

22. S. Jonsson and M. O. Karlsson. Estimation of dosing strategies aiming at maximizing utility or responder probability, using oxybutynin as an example drug. Eur. J. Pharm. Sci. 25:123-132 (2005).

23. L. B. Sheiner and K. L. Melmon. The utility function of antihypertensive therapy. Ann. N.Y. Acad. Sci. 304:112-127 (1978).

24. E. Zanderigo, V. Sartori, G. Sveticic, T. Bouillon, P. Schumacher, M. Morari, and M. Curatolo. The well-being model: a new drug interaction model for positive and negative effects. Anesthesiology 104:742-753 (2006).

25. S. L. Beal, L. B. Sheiner. NONMEM user's guide. NONMEM Project Group, University of California at San Fransisco, San Fransisco, 1999.

26. D. R. Mould, G. F. Fleming, K. M. Darcy, and D. Spriggs. Population analysis of a 24-h paclitaxel infusion in advanced endometrial cancer: a gynaecological oncology group study. $B r$. J. Clin. Pharmacol. 62:56-70 (2006).

27. L. B. Sheiner, D. R. Stanski, S. Vozeh, R. D. Miller, and J. Ham. Simultaneous modeling of pharmacokinetics and pharmacodynamics: application to $d$-tubocurarine. Clin. Pharmacol. Ther. 25:358-371 (1979).

28. M. Cullberg, U. G. Eriksson, K. Wahlander, H. Eriksson, S. Schulman, and M. O. Karlsson. Pharmacokinetics of ximelagatran and relationship to clinical response in acute deep vein thrombosis. Clin. Pharmacol. Ther. 77:279-290 (2005).

29. S. Ko, D. H. Goldstein, and E. G. VanDenKerkhof. Definitions of "respiratory depression" with intrathecal morphine postoperative analgesia: a review of the literature. Can. J Anaesth. 50:679-688 (2003).

30. D. Grace, H. Bunting, K. R. Milligan, and J. P. Fee. Postoperative analgesia after co-administration of clonidine and mor- phine by the intrathecal route in patients undergoing hip replacement. Anesth. Analg. 80:86-91 (1995).

31. L. Jacobson, C. Chabal, M. C. Brody, R. J. Ward, and R. C. Ireton. Intrathecal methadone and morphine for postoperative analgesia: a comparison of the efficacy, duration, and side effects. Anesthesiology 70:742-746 (1989).

32. L. L. Gustafsson, B. Schildt, and K. Jacobsen. Adverse effects of extradural and intrathecal opiates: report of a nationwide survey in Sweden. Br. J. Anaesth. 54:479-486 (1982).

33. A. Dahan, J. DeGoede, A. Berkenbosch, and I. C. Olievier. The influence of oxygen on the ventilatory response to carbon dioxide in man. J. Physiol. 428:485-499 (1990).

34. R. Romberg, E. Olofsen, E. Sarton, L. Teppema, and A. Dahan. Pharmacodynamic effect of morphine-6-glucuronide versus morphine on hypoxic and hypercapnic breathing in healthy volunteers. Anesthesiology 99:788-798 (2003).

35. L. S. Brady and S. G. Holtzman. Analgesic effects of intraventricular morphine and enkephalins in nondependent and morphine-dependent rats. J. Pharmacol. Exp. Ther. 222:190-197 (1982).

36. A. J. Clark. General Pharmacology. In Heffner's Handbuch der experimentellen Pharmacologie Erganzungsband, Band 4, Springer, Berlin, 1937, pp. 38-51.

37. A. Dahan, A. Yassen, R. Romberg, E. Sarton, L. Teppema, E. Olofsen, and M. Danhof. Buprenorphine induces ceiling in respiratory depression but not in analgesia. Br. J. Anaesth. (2006).

38. S. L. Walsh, K. L. Preston, M. L. Stitzer, E. J. Cone, and G. E. Bigelow. Clinical pharmacology of buprenorphine: ceiling effects at high doses. Clin. Pharmacol. Ther. 55:569-580 (1994).

39. T. Kenakin. Pharmacologic analysis of drug-receptor interaction, Lippincott-Raven, New York, 1997.

40. A. Yassen, E. Olofsen, J. Kan, A. Dahan, and M. Danhof. Animal-to-human extrapolation of the pharmacokinetic and pharmacodynamic properties of buprenorphine. Clin. Pharmacokinet. 46:433-447 (2007).

41. C. Regnard and A. Pelham. Severe respiratory depression and sedation with transdermal fentanyl: four case studies. Palliat. Med. 17:714-716 (2003).

42. K. A. Sporer. Buprenorphine: a primer for emergency physicians. Ann. Emerg. Med. 43:580-584 (2004). 